\title{
A Population Decoding Framework for Motion Aftereffects on Smooth Pursuit Eye Movements
}

\author{
Justin L. Gardner, Stefanie N. Tokiyama, and Stephen G. Lisberger \\ Howard Hughes Medical Institute, Bioengineering Graduate Group, W. M. Keck Foundation Center for Integrative Neuroscience, and Department of \\ Physiology, University of California, San Francisco, California 94143
}

\begin{abstract}
Both perceptual and motor systems must decode visual information from the distributed activity of large populations of cortical neurons. We have sought a common framework for understanding decoding strategies for visually guided movement and perception by asking whether the strong motion aftereffects seen in the perceptual domain lead to similar expressions in motor output. We found that motion adaptation indeed has strong sequelae in the direction and speed of smooth pursuit eye movements. After adaptation with a stimulus that moves in a given direction for $7 \mathrm{sec}$, the direction of pursuit is repelled from the direction of pursuit targets that move within $90^{\circ}$ of the adapting direction. The speed of pursuit decreases for targets that move at the direction and speed of the adapting stimulus and is repelled from the adapting speed in the sense that the decrease either becomes greater or smaller (eventually turning to an increase) when tracking targets move slower or faster than the adapting speed. The effects of adaptation are spatially specific and fixed to the retinal location of the adapting stimulus. The magnitude of adaptation of pursuit speed and direction is uncorrelated, suggesting that the two parameters are decoded independently. Computer simulation of motion adaptation in the middle temporal visual area (MT) shows that vector-averaging decoding of the population response in MT can account for the effects of adaptation on the direction of pursuit. Our results suggest a unified framework for thinking, in terms of population decoding, about motion adaptation for both perception and action.
\end{abstract}

Key words: adaptation; MT; visual motion processing; waterfall illusion; distributed representations; vector averaging

\section{Introduction}

The brain relies on the activity of large populations of neurons to form a perception or program a movement. The mechanism that decodes neural population responses is a critical link for understanding how both perceptual judgments and motor commands are generated. Yet, perception and action make different demands of visual information and consequently exhibit some dissimilarities in processing. For example, a variety of perceptual visual illusions are not found for visually guided movement systems, which require veridical information about the environment (Goodale and Milner, 1992). Motion aftereffects are a well known class of visual illusions that have been used as a highly diagnostic means of inferring information about visual processing for perception. However, it is not known whether they are present for visual motion-guided movement, let alone whether they are similar for motor systems and perception (Wohlgemuth, 1911; Mather et al., 1998). We tested for motion aftereffects on a

Received Jan. 30, 2004; revised Sept. 2, 2004; accepted Sept. 3, 2004.

This work was supported by National Institutes of Health Grant EY03878 and the Howard Hughes Medical Institute. J.L.G. was supported by a Burroughs Wellcome Fund training grant in quantitative biology and a postdoctoral fellowship from the Japan Society for the Promotion of Science. We thank Scott Ruffner for computer programming, Ken McGary for electronics, and Laszlo Bocskai for machining. We also thank Karen MacLeod and Elizabeth Montgomery for attention to the welfare of the monkey subjects, I-han Chou and Adam Kohn for helpful discussions, and Hilary Heuer for comments on a previous version of this manuscript.

Correspondence should be addressed to Dr. Justin L. Gardner, Department of Psychology, New York University, 6 Washington Place, 8th Floor, New York, NY 10003. E-mail: justin@cns.nyu.edu.

DOl:10.1523/JNEUROSCI.0337-04.2004

Copyright $\odot 2004$ Society for Neuroscience $\quad$ 0270-6474/04/249035-14\$15.00/0 visually guided movement system as a way to understand neural population decoding for perception and action.

In perception, the classic motion aftereffect is a visual illusion whereby long adaptation to a moving pattern leads to the strong perception that stationary objects are moving opposite to the adapting motion (Purkinje, 1820; Addams, 1834). Similar adaptation causes perceptual judgments of target direction to be repelled from the adapting direction when the actual target motion is in a direction that is close to, but not exactly, the adapting direction (Levinson and Sekuler, 1976; Schrater and Simoncelli, 1998). If motor output displayed the same adaptation effects as perception, then we could conclude that adaptation operates at a processing level that contributes to both action and perception. Furthermore, we then could use the motor output, with its advantages for relating neural activity to behavior, as a tool for understanding population decoding and adaptation for both perception and action.

We have used visual motion as a stimulus, perturbed the population code for motion with motion adaptation, and explored the consequences for smooth pursuit eye movements. Our analysis exploited the well established link between visual motion physiology and pursuit (Lisberger et al., 1987). Neurons in the middle temporal (MT) visual area are selectively sensitive to visual motion (Zeki, 1974; Maunsell and Van Essen, 1983; Albright, 1984) and provide signals that are used to determine the speed and direction of pursuit (Newsome et al., 1985; Dursteler and Wurtz, 1988; Komatsu and Wurtz, 1989). Analysis of the relationship between neural population activity for target speed and 
pursuit (Churchland and Lisberger, 2001; Priebe and Lisbeger, 2004) has implied that the decoding computation implements a subtle modification of the well known and neurally plausible computation known as "vector averaging" (Georgopoulos et al., 1986; Lee et al., 1988). However, little is known about decoding the population code for target direction or about the relationship between decoding computations for direction and speed.

Our results provide evidence of similar principles underlying population decoding for perception and action. They show that motion adaptation causes strong and reliable aftereffects on pursuit direction and speed. The aftereffects can be understood in a framework based on decoding the population response of MT, where distinct decoding computations are used to extract the direction and speed of target motion.

\section{Materials and Methods}

Our database of over 64,000 trials came from 130 different experimental sessions conducted on three adult male rhesus monkeys (Macaca mulatta), $10-13 \mathrm{~kg}$ in weight. All methods had received previous approval from the Institutional Animal Care and Use Committee at the University of California, San Francisco, and conformed to the National Institutes of Health Guide for the Care and Use of Laboratory Animals. Behavioral training, general experimental procedures, and surgical procedures have been described in detail previously (Lisberger and Westbrook, 1985). Monkeys were trained initially to sit in a primate chair and respond to dimming of spots of light for liquid reinforcement. After training, a head holder was implanted over the skull using 6-mm-wide orthopedic stainless steel strips, 6- and 8-mm-long screws, and dental acrylic. A scleral search coil used to measure eye position was sutured to the sclera a few weeks later using a variation of a technique devised by Judge et al. (1980). All surgeries were performed using sterile procedures while the monkey was under general anesthesia with inhaled isofluorane. Analgesic treatments with buprenorphine $\mathrm{HCl}(0.01-0.03 \mathrm{mg} / \mathrm{kg})$ and ketorolac (7.5-15 mg) were administered postoperatively for several days. After recovery from surgery, the monkeys were positioned in front of a display screen with their head holders secured to the ceiling of the chair and were trained to fixate and track stationary and/or moving visual targets.

Experimental and stimulus control. Visual stimuli were presented on a Hewlett-Packard 1304A analog oscilloscope that was placed $28.5 \mathrm{~cm}$ away from the monkey's eyes and subtended $48 \times 42^{\circ}$ of visual angle. The screen was completely updated every $4 \mathrm{msec}$ for a refresh rate of $250 \mathrm{~Hz}$. The horizontal and vertical positions of the display beam of the oscilloscope were driven by 16 bit digital-to-analog converters on a digital signal processing board, affording 64,000 dot positions across the screen in each direction. Adapting and control stimuli were patches of randomly placed dots at an average density of 1 dot per $1.44^{\circ}$ squared. Each dot had a luminance of $1.7 \mathrm{~cd} / \mathrm{m}^{2}$. Pursuit targets and fixation spots consisted of $0.4^{\circ}$ spots of light and also had a luminance of $1.7 \mathrm{~cd} / \mathrm{m}^{2}$. All experiments were conducted in a reasonably dark room with the only sources of illumination coming from the display oscilloscope.

Experiments were controlled by a DEC Alpha Unix workstation running a customized computer application and a $500 \mathrm{MHz}$ Pentium personal computer running Windows NT and VenturCom RTX. Commands were sent from the Unix workstation to the personal computer, which provided real-time control of the visual stimuli and data acquisition. Eye velocity was obtained by passing the eye position voltages through an analog circuit that differentiated frequencies up to $25 \mathrm{~Hz}$ and rejected higher frequencies with a roll-off of $20 \mathrm{~dB} /$ decade. Signals proportional to eye position and velocity were digitized at $1 \mathrm{kHz}$ on each channel and stored for subsequent analysis.

Experimental design. Experiments were based on a trial configuration that contained the sequence of stimuli diagrammed in Figure 1: (1) Fixation: monkeys were required to fixate a central point within $2^{\circ}$ for 600 msec. (2) Adapt: a motion adapting patch was presented for $7-7.5 \mathrm{sec}$ while the monkey continued to fixate. The adapting patch was square. For most of our experiments, it was $10 \times 10^{\circ}$ in size and was centered $6^{\circ}$ from the fixation point, as illustrated in Figure 1. For central adaptation trials, the patch was $5 \times 5^{\circ}$ in size and was centered at the fixation point. The dots within the patch either were stationary or moved coherently at $16 \%$ sec within a stationary, invisible aperture. (3) Fixation: the adapting patch was extinguished, and the monkey continued to fixate for $400-500$ msec. (4) Pursuit: the fixation point was extinguished, and a pursuit target appeared at an eccentric location and moved at $16 \%$ sec for 625 msec, traversing a distance of $10^{\circ}$. Once the target started moving, the monkey was given $350 \mathrm{msec}$ to capture it and then was required to maintain gaze within $3.5^{\circ}$ of the moving target for the duration of its motion. (5) Fixation: the target remained visible when it stopped moving, and the monkey was required to fixate for another $200 \mathrm{msec}$.

Liquid reward was given periodically during the 7-7.5 sec adapting period. A much larger volume of liquid was given for successful completion of the trial. A trial was aborted if the monkey failed to keep gaze within the fixation accuracy window. The amount and timing of the liquid rewards given during fixation and at the end of the trial were varied on a day-by-day basis to prevent the monkey from adopting a strategy of maintaining fixation during the adapting period but from aborting the trial before the pursuit portion was completed. Anticipatory movements (Dodge, 1903; Kowler and Steinman, 1979; Barnes and Asselman, 1991) were minimized by three methods: (1) the interval between offset of the adapting patch and onset of the pursuit target was randomized; (2) the direction of motion of the pursuit target was randomized; and (3) pursuit targets were presented randomly in either visual hemifield.

The initial position of the pursuit target was programmed such that it would intersect the middle of the adapting patch $250 \mathrm{msec}$ after motion onset, and the direction was varied according to the exact goal of each experiment. For some starting positions, the distance the target traveled had to be shortened because the oscilloscope was not large enough to accommodate a full $10^{\circ}$ of traversal from the chosen starting position. The location of the adapting patch was counterbalanced across experimental sets to be either in the left or right visual hemifield, and adapting motion and pursuit targets could move either toward or away from the fixation position. Because no systematic differences were found for these different conditions, we present analysis of these data together in the text. Every experimental block contained pursuit targets originating in both visual hemifields.

A daily experiment usually consisted of a control block of trials with stationary dots in the adapting patch, followed by an adaptation block with moving dots. On some occasions, we reversed the order of blocks, without any consistent changes in the results. Between control and adaptation blocks, the room was illuminated with overhead incandescent lamps, and the monkey was allowed to view freely for several minutes, to minimize any storage effects of adaptation (Wohlgemuth, 1911; Spigel, 1960; Thompson and Wright, 1994; Verstraten et al., 1994). Unless noted otherwise, all experimental paradigms in this report were tested on at least two different monkeys with no systematic difference between monkeys. Because of the consistency across monkeys, we will report only the number of experiments done in each condition.

Data analysis. In each trial, semiautomated software marked the onset and offset of the first and second saccade after the start of target motion. The automatic algorithm identified the two time points when radial eye velocity rose above and subsequently fell below $50 \% \mathrm{sec}$. Saccade onset and offset were marked at $10 \mathrm{msec}$ before the first time point and $20 \mathrm{msec}$ after the second time point, times determined empirically to give excellent results on the majority of trials. Subsequent visual inspection of all trials confirmed in most trials that the marks excluded eye velocity attributable to saccades with as little loss of pursuit eye velocity data as possible. In cases in which visual inspection deemed the marks to be inaccurate, the operator cleared the marks and replaced them by hand. At this stage of analysis, we also discarded any trials in which eye movement traces showed slow oscillations indicative of sleepiness on the part of the monkey.

Our pursuit targets were designed to cross the center of the location where the adapting patch had been $250 \mathrm{msec}$ after motion onset. Therefore, we could not adhere to the usual strategy of starting the motion of pursuit targets at locations customized to eliminate saccades (Lisberger et al., 1987), and our monkeys almost always made catch-up saccades to align the position of the fovea with the target. Because the early saccades 
made it impossible to analyze pre-saccadic pursuit eye velocity, we have instead used the eye velocity in the first $10 \mathrm{msec}$ after the end of the first saccade to analyze the effect of motion adaptation on the initiation of pursuit. Post-saccadic eye velocity is a valid measure because it depends on the image motion present before the saccade (Chou and Lisberger, 2002), which we could assess and control. It is a preferred measure for experiments that use a range of starting positions for pursuit targets because, unlike pre-saccadic pursuit (Lisberger and Westbrook, 1985), post-saccadic smooth eye velocity does not depend on the initial position of the pursuit target and is not subject to asymmetries that give rise to better initiation of pursuit for targets moving toward versus away from the position of fixation (Lisberger, 1998).

We also averaged eye velocity in $10 \mathrm{msec}$ bins starting from the onset of motion of the pursuit target. For the latter analysis, bins that contained a saccade were excluded from the average. In general, we collected 20 repeats of any particular trial type for both the control and adaptation blocks. Distributions of directions of pursuit responses were compared using the Watson-Williams test (Batschelet, 1981). Eye speeds were expressed as a percentage of target speed, and distributions were compared using log-transformed Student's $t$ test. Bootstrapping (Mooney and Duval, 1993) was used to evaluate statistical significance of results when parametric tests were either not available or not suitable. Bootstrapping of statistics was accomplished by randomly resampling the original distribution with replacement 1000 times and recalculating the statistic of interest. We estimated the $95 \%$ confidence intervals by reporting the 25th smallest and largest value of the statistic generated. All data analyses were performed using Matlab (Mathworks, Natick, MA).

Because of the large duration of presentation of the adapting patch, the analysis of the quality of fixation required a slightly modified approach. We set the velocity criteria for detecting saccades to $8 \%$ sec to detect the smaller saccades present during fixation. Visual inspection of $10 \%$ of the trials verified that the lower threshold did not cause us to mistake rapid smooth eye movements for saccades. Furthermore, graphs of the maximum velocity versus the amplitude of the saccades detected during fixation revealed a linear relationship with a slope of $41.3 \%$ sec per degree $(p<0.001)$, consistent with the main sequence of saccades (Bahill et al., 1975). Saccades detected during fixation had an amplitude of $0.66 \pm$ $0.47^{\circ}(\mathrm{SD})$ and occurred at an average frequency of 2.11 saccades per second.

Computer simulations. We fitted our results with a model of the effects of adaptation on the direction tuning of MT neurons. The response of an MT neuron under control conditions was expressed as the product of two Gaussian functions:

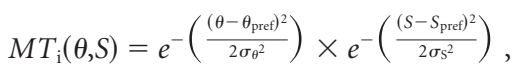

where $\theta$ and $S$ are the direction and speed of target motion, $\theta_{\text {pref }}$ and $S_{\text {pref }}$ are the preferred direction and speed of the neuron, and $\sigma_{\theta}$ and $\sigma_{\mathrm{s}}$ are the widths of the tuning for direction and speed, respectively. Speed was expressed in units of $\log$ base two. Preferred directions of the model MT neurons were chosen at 16 equally spaced points from 0 to $360^{\circ}$ (not including $360^{\circ}$ ), and preferred speeds were chosen at 16 equally spaced velocities (on a $\log$ axis) from $1 / 256-1024^{\circ} / \mathrm{sec}$ (i.e., -8 to 10 on a log base two axis). We constructed one model MT neuron for each combination of preferred speed and direction for a total of 256 model neurons. Directional tuning widths were set to $45^{\circ}$ for a full-width at half-height of $106^{\circ}$, close to the value from the literature for neurons studied with single dots (Albright, 1984). Speed-tuning widths were set to one octave, also in the range of tuning widths found in the literature (Maunsell and Van Essen, 1983; Rodman and Albright, 1987). We obtained quantitatively identical results if we used only 16 model neurons of a single preferred speed and qualitatively similar results if we used a larger number of model neurons $(n=10,000)$ with direction and speed preferences chosen randomly from a uniform distribution. The results of the 10,000 neuron simulations are used to illustrate the change in population response in Figure 9, but model fits were all done with a simulation that comprised 256 model neurons with equally spaced tuning preferences.

We modeled four different types of possible adaptation effects on the direction tuning of MT neurons: (1) a multiplicative gain change; (2) narrowing of the tuning width; (3) repellant; and (4) attractive changes in the preferred tuning of neurons. For the multiplicative gain change model, the response of an MT neuron after adaptation was expressed as the product of the $M T_{\mathrm{i}}(\theta, S)$ from Equation 1 and an inverted Gaussian function:

$$
\begin{aligned}
& M T_{\mathrm{i}, \text { adapted }}(\theta, S)=\left(1-\left(1-A_{\text {adapt }}\right) \times e^{\left.-\left(\frac{\left(\theta_{\text {pref }}-\theta_{\text {adap }}\right)^{2}}{2 \sigma_{\text {adapt }}{ }^{2}}\right)\right)}\right. \\
& \times M T_{\mathrm{i}, \text { control }}(\theta, S) .
\end{aligned}
$$

The maximum reduction of gain had an amplitude of $A_{\text {adapt }}$ at the adapting direction $\left(\theta_{\text {adapt }}\right)$, and $\sigma_{\text {adapt }}$ was the SD of the adaptation Gaussian. We modeled the narrowing of direction-tuning bandwidth in a similar manner, by first changing the directional-tuning width for every neuron according to the following equation:

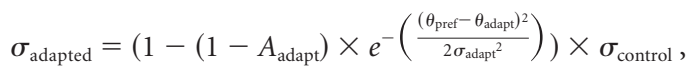

and then recalculating the adapted responses with the new tuning widths. To shift the tuning preferences, we first calculated the magnitude of shift of preferred tuning according to a Gaussian function:

$$
\text { shift }=A_{\text {adapt }} \times e^{-\left(\frac{\left(\left|\theta_{\text {pref }}-\theta_{\text {adapt }}\right|-\theta_{\text {shifit }}{ }^{2}\right.}{2 \sigma_{\text {adapt }}{ }^{2}}\right),}
$$

where $\theta_{\text {adapt }}$ was not a model parameter but the actual direction of adapting motion, and $\theta_{\text {shift }}$ was the difference in direction preference from the adapting direction $\left(\theta_{\text {adapt }}\right)$ that experienced the maximum shift $\left(A_{\text {adapt }}\right)$. We then shifted the tuning preference either toward (attraction model) or away from (repellant model) the adapting direction accordingly. Model neurons with preferred tuning before adaptation that was the same as the adapting direction did not change their preference. For the attraction model, if the shift in tuning would have caused the preferred tuning direction to shift past the adapting direction, then the tuning preference of the neurons was set to the adapting direction.

We used the responses from the model neurons and their preferred directions and speeds to calculate the population response. The response of each model neuron was considered as a vector in the preferred direction for that neuron, with a length equal to the response of the neuron as calculated by the equations above. The population direction was calculated by performing a vector average of all the individual model neuron responses, defined as the vector sum of all the response vectors of a neuron divided by the total population response:

$$
\text { Population direction estimate }(\theta, s)=\frac{\sum_{i=1}^{n} \vec{R}_{R}\left(\theta_{\text {pref }_{\mathrm{i}}}, M T_{\mathrm{i}}(\theta, S)\right)}{\sum_{i=1}^{n} M T_{\mathrm{i}}(\theta, S)} \text {. }
$$

where, $\vec{R}\left(\theta_{\text {pref }_{\mathrm{i}}}, M T_{\mathrm{i}}(\theta, S)\right)$ is the vector response of the $i$ th model neuron to the stimulus with direction $\theta$ and speed $S$. To calculate the population speed estimate, we used the following equation:

$$
\text { Population speed estimate }(\theta, s)=g(\varepsilon) \times \frac{\sum_{i=1}^{n} S_{\text {pref }_{\mathrm{i}}} \times M T_{\mathrm{i}}(\theta, S)}{\varepsilon+\sum_{i=1}^{n} M T_{\mathrm{i}}(\theta, S)} \text {, }
$$

where the summations are over all neurons and $\epsilon$ biases the speed estimate toward zero when the population response is small. We note that an opponent motion computation to compare the responses of neurons with opposite preferred directions is a critical feature of computations that decode target speed for apparent motion targets (Churchland and 
Lisberger, 2001). Because (1) adaptation has little or no effect on the response on neurons that prefer the opposite direction relative to the adapting motion (Petersen et al., 1985; Van Wezel and Britten, 2002; Kohn and Movshon, 2003) and (2) our pursuit targets are not expected to evoke significant opponent responses from neurons tuned to the opposite direction, it was not necessary to include an opponent calculation in Equation 6. The gain factor $(g(\epsilon))$ in Equation 6 was used to make the model produce a veridical speed before adaptation for targets moving at the adapting speed of $16^{\circ} / \mathrm{sec}$. The gain factor was computed as follows:

$$
g(\varepsilon)=\frac{\varepsilon+\sum_{i=1}^{n} M T_{\mathrm{i}}}{\sum_{i=1}^{n} M T_{\mathrm{i}}}
$$

where $M T_{\mathrm{i}}$ is the response of the $i$ th MT neuron for pursuit targets of the speed and direction of the adapting stimulus before adaptation. To an extent that depends on the value of $\epsilon$, the model will underestimate the speed of targets moving much slower than the adapting speed.

We fitted the model to our data for the effect of adaptation on pursuit of subsequent target motion of different directions. Nonlinear leastsquares fitting with the Levenberg-Marquardt algorithm (for reference, see Press et al., 1988) was used to find adaptation parameters that optimized the agreement between the directions and speeds of smooth eye motion predicted by the model and those measured in the data. We repeated the fitting procedure 10 times starting with different random values of the parameters and chose as optimized parameters those that produced the least squared difference between the outputs from the model and the data.

\section{Results}

Figure $1 B$ shows an example of the kind of effect we analyzed. After the pursuit target started to move at a constant velocity, the monkey initiated pursuit weakly, made a saccade to bring eye position close to target position, and showed excellent pursuit immediately after the saccade. Before the saccade, eye velocity during the adaptation block (gray trace) was considerably lower than it had been during the preceding control block (black trace). After the saccade, horizontal eye speed was close to target speed in both blocks but was consistently larger in the control block than in the adaptation block. Figure $1 B$ illustrates one small facet of our data set: the effect of adaptation with rightward target motion on the speed of the horizontal component of the response to rightward motion. Most of the rest of this report shows how this effect is expressed in terms of the direction and speed of pursuit responses to target motions in different directions relative to that of the adapting motion.

\section{Adaptation of the direction and speed of pursuit}

Effects of adaptation on the direction of pursuit appeared when we included pursuit target motion in directions that were different from that of the adapting patch. Figure $2 \mathrm{~A}$ schematizes an experiment in which the adapting motion was rightward and pursuit target motions were in polar directions that were 30 or $60^{\circ}$ upward and downward relative to rightward. The results of this experiment are summarized in the polar plot of Figure $2 C$, where each line is a vector showing the direction and speed of post-saccadic pursuit. Comparison of the average vectors of pursuit in the control block (bold, black lines) and the vectors for individual trials in the adaptation block (colored vectors) reveals two effects. First, the direction of pursuit in individual responses during the adaptation blocks (colored vectors) is consistently repelled away from the direction of the adapting stimulus. For
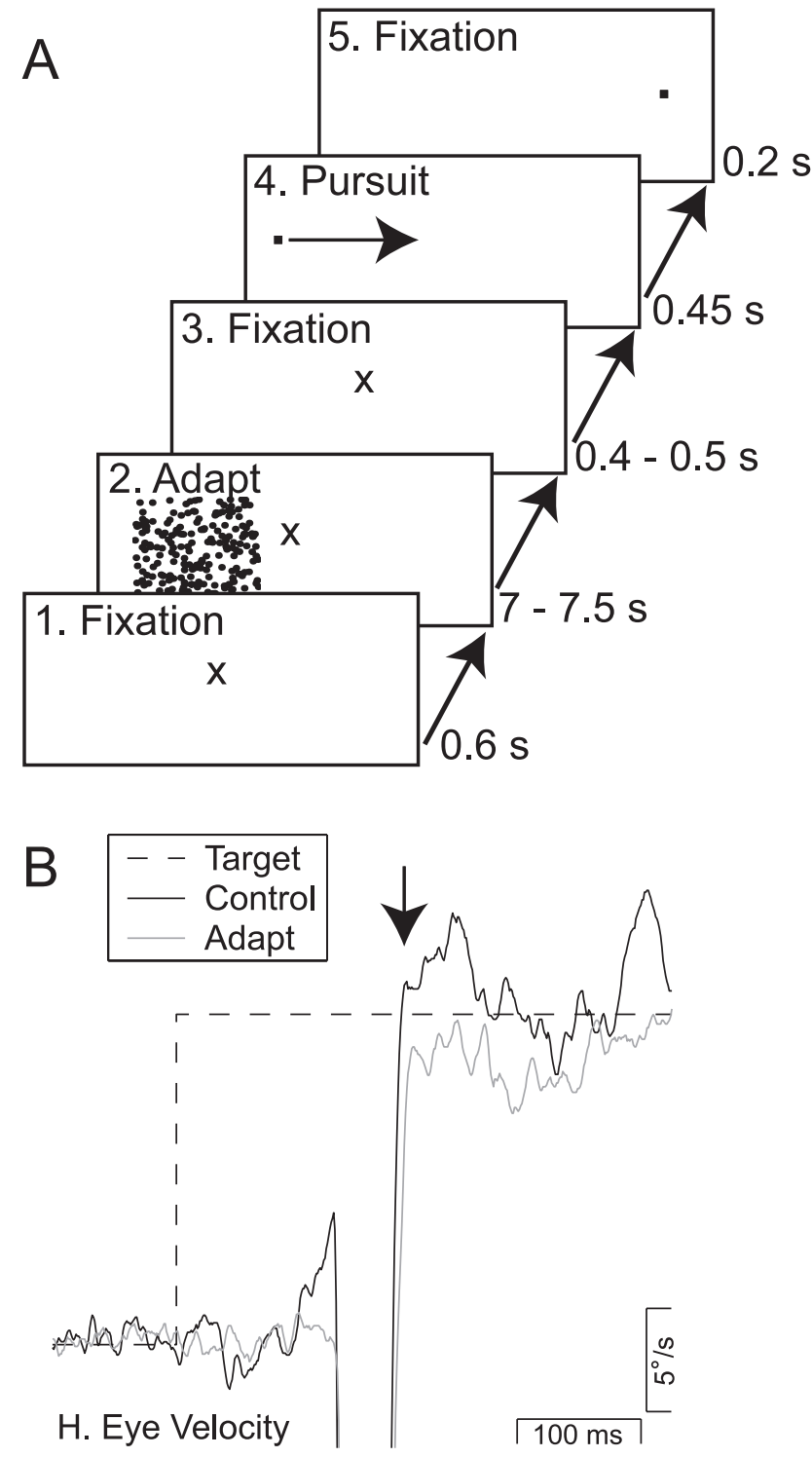

Figure 1. Schematic diagram of an example trial configuration and representative eye velocity data traces. $A$, The screen shots show the steps in target presentation, moving from bottom left to top right: (1) Fixation: the " $x$ " shows a fixation point at the center of the screen; (2) Adapt: the " $x$ " shows the persistent fixation point, and the patch of dots indicates the adapting stimulus; (3) Fixation: the " $x$ " again shows the fixation point; (4) Pursuit: the dot shows the pursuit target, and the arrow shows its motion; (5) Fixation: the dot is the final fixation target, now moved to the edge of the screen where the pursuit target stopped. $B$, Example eye velocity traces for one trial. The dashed trace shows target velocity, and the black and gray traces show horizontal eye velocity in the absence and presence of a previous adapting stimulus. The downward arrow shows the onset of the $10 \mathrm{msec}$ post-saccadic measurement interval. Upward deflections represent rightward motion.

target motions in polar directions of -30 and $-60^{\circ}$, the responses in the adaptation block are rotated clockwise relative to the average from the control block. The average directions moved to $-52.7^{\circ}$ from $-41.1^{\circ}$ and to $-26.4^{\circ}$ from $-20.8^{\circ}(p<0.02$; Watson-Williams test). For target motions in polar directions of 30 and $60^{\circ}$, adapted responses were rotated counterclockwise to $38.0^{\circ}$ from $33.0^{\circ}$ and to $64.3^{\circ}$ from $54.8^{\circ}$ ( $p<0.01$; WatsonWilliams test). Second, the speed of post-saccadic pursuit tends to be lower in the adaptation blocks than in the control blocks. The median eye speeds for the four directions of pursuit motion were adapted to $12.5 \% \mathrm{sec}$ from $12.9 \% \mathrm{sec}$, to $9.2 \% \mathrm{sec}$ from $16.4 \%$ 

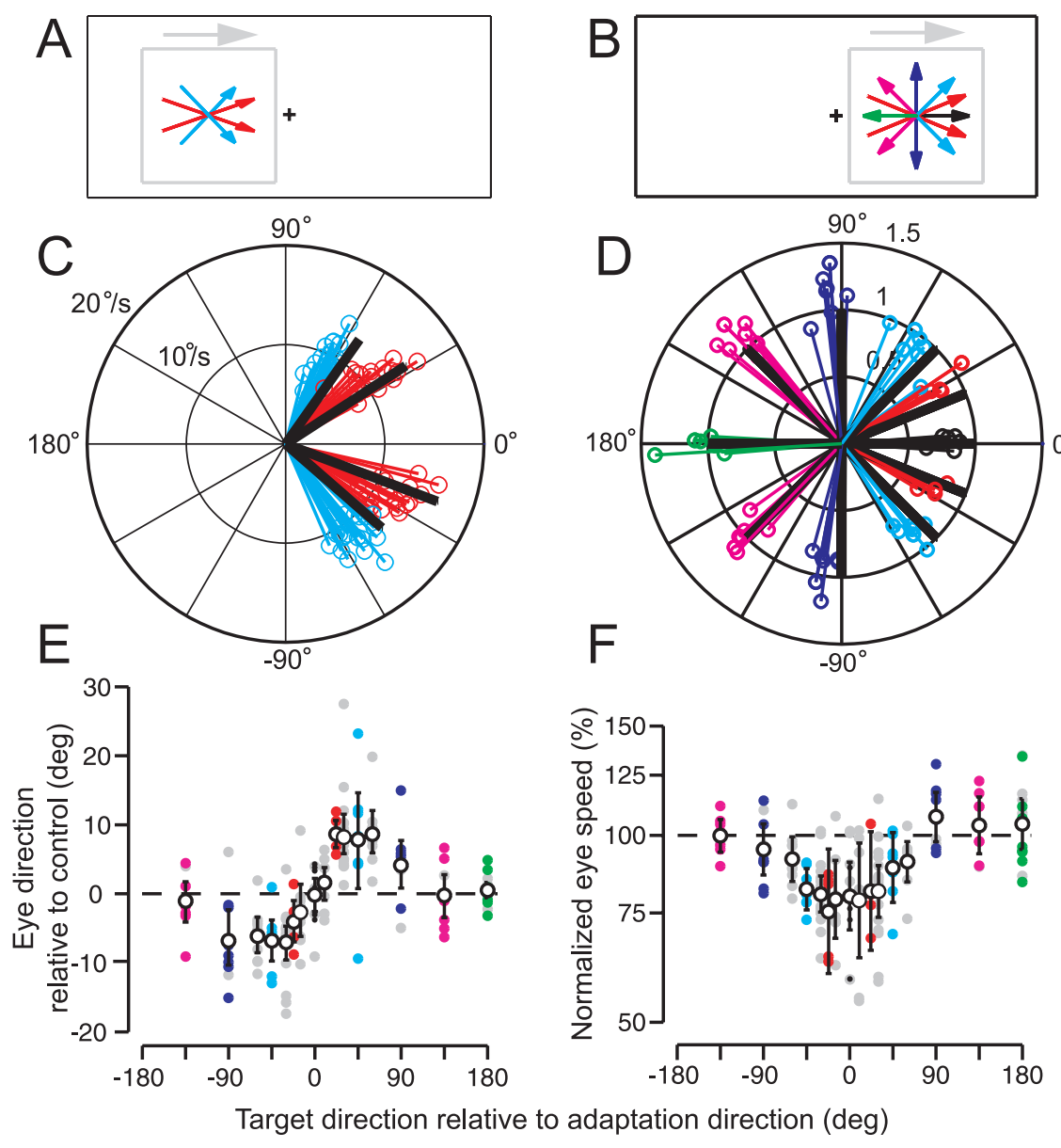

Figure 2. The effect of adaptation on subsequent pursuit, and how adaptation generalizes to targets moving in different directions. The right and left panel summarize two sets of experiments that used different directions of pursuit target motion to explore the effects of adaptation. $A, B$, Schematic diagram of the target motions. The light gray outline shows the location of the adapting patch, the light gray arrow shows the direction of the adapting motion, and the " + " indicates the fixation position during adaptation. The colored arrows show the trajectory of the first $500 \mathrm{msec}$ of motion of the pursuit targets. C, D, Polar plots where each arrow is a vector showing the direction and amplitude of eye velocity. The colors of the vectors correspond to the target motion vectors in $A$ and $B$. In $C$, the bold black vectors indicate the average responses in control blocks, without adaptation, and the colored vectors indicate responses in individual trials in the adaptation block. In $D$, all the responses in the adaptation block have been normalized relative to the average responses in the control block so that the bold black vectors are in the directions of target motion with unity length. The colored vectors show the normalized average responses in each of seven experiments. $E, F$, Summary of all 29 experiments plotting normalized eye direction $(E)$ and speed $(F)$ as a function of the difference between the direction of motion of the pursuit target and the adapting stimulus. The colored symbols plot the data from $D$, the gray symbols plot the results for 22 additional experiments, and the open circles plot the averages across all experiments. Error bars show the $95 \%$ confidence intervals.

sec, to $10.2 \% \mathrm{sec}$ from $14.4 \% \mathrm{sec}$, and to $11.1 \% \mathrm{sec}$ from $12.9 \% \mathrm{sec}$ (all $p<0.01$; log-transformed Student's $t$ test).

An experiment that used a larger number of directions of pursuit target motion is summarized in Figure 2, $B$ and $D$, now using a data presentation that normalizes the responses in the adaptation block relative to those in the control block. Pursuit was tested for target motions in polar directions $0, \pm 22.5, \pm 45$, $\pm 90, \pm 135$, and $180^{\circ}$. For each response in the adaptation block, eye speed has been normalized for the average eye speed in the control block, and eye direction has been computed relative to the average direction of the responses in the control block. As a result, the average responses in the control block (bold, black vectors) have lengths of unity and directions equal to target direction. Figure $2 D$ plots seven vectors of each color, to show the average responses in the adaptation block for seven experiments conducted with the design illustrated in Figure $2 B$. In each exper- iment, the repellant effects of adaptation extended to directions of target motion up to $90^{\circ}$ away from the adapting direction (all differences $p<0.03$ and $p<0.01$ for target motion with upward and downward components; Watson-Williams test). In contrast, the pursuit directions in the adaptation block were not significantly different from those in the control block for target motion in polar directions 180,135 , -135 , and $0^{\circ}(p>0.2$; Watson-Willams test). Adaptation reduced the speed of pursuit for directions close to the adapting direction with significant differences for polar directions of $0,-22.5$, and -45 $(p<0.01 ;$ log-transformed Student's $t$ test); pursuit speed was not affected for other directions of target motion. Although we have represented the position and direction of the adapting patch to be on the right with motion to the right in Figure $2 B$, the seven experiments summarized in Figure $2 D$ included all possible combinations of patch locations either on the left or right side of the fixation position with directions of adapting motion either to the left or right. Pursuit targets always were presented on the same side of the fixation position as the adapting patch. Because there were no consistent differences in the amount of reduction in speed or spread of the repellant effect to different directions of pursuit, the results have been rotated so that they could be presented as if the adapting motion were rightward.

All 29 adaptation experiments of the designs similar to those illustrated in Figure $2, A$ and $B$ ( $n=7$ for the full set of directions as in $B ; n=22$ that examined fewer directions), are summarized by showing the normalized eye direction and speed in Figure 2, $E$ and $F$, as a function of the difference between the polar direction of pursuit target motion and that of the adapting stimulus. The circular symbols with error bars show means and 95\% confidence intervals for the full set of experiments, whereas the gray and colored dots show the average normalized effects of adaptation based on $\sim 20$ pursuit responses in each direction. The direction of pursuit (Fig. $2 E$ ) is clearly repelled from the direction of the adapting stimulus, showing positive effects for positive directions of target motion and negative effects for negative directions of target motion. The average effect on pursuit direction peaked for polar directions $\pm 30-60^{\circ}$ from the adapting direction, and statistical significance was present for pursuit target motions up to $90^{\circ}$ from the adapting direction. The speed of pursuit (Fig. $2 F$ ) is maximally depressed for pursuit target motion in the adapting direction and retains statistical significance for pursuit target directions $<90^{\circ}$ from the adapting direction. We did not observe significant enhancement of pursuit speed when the pursuit target moved in directions opposite to the adapting direction.

To determine limits on how quickly adaptation can be in- 
duced by our experimental design, we conducted four experiments that consisted of a control block, an adaptation block with a reduced duration of exposure of 1-1.5 sec for each adapting stimulus, and a second control block and adaptation block with our standard exposure time of $7-7.5 \mathrm{sec}$. The experiments had the same design as the one diagrammed in Figure $2 A$, with four directions of motion for the pursuit target. The pursuit in both adaptation blocks was normalized to their respective control blocks. The adaptation of pursuit direction was statistically significant for the shorter adapting stimuli but not as strong as for the longer adapting stimuli. For the shorter-duration adapting stimulus, the effects on pursuit direction averaged 59\% of those for the longer-duration adapting stimulus. There were no differences in the extent of adaptation of pursuit speed, which averaged 84.1 and $85.2 \%$ of the control response for the short- and longduration adapting stimuli.

We also conducted three experiments on one monkey to ask whether the effects of adaptation were stored from trial to trial: would control and adaptation trials in separate blocks show stronger effects of adaptation than if the trials were interleaved? Each experiment consisted of a "mixed" block that contained randomly interleaved control and adaptation trials as well as a standard adaptation block, both using our standard adaptation period of 7-7.5 sec. The effects of adaptation in the standard adaptation block were compared with the control trials in the mixed block. We found that the direction of pursuit was weakly repelled from the adapting direction in the mixed block: the effects on pursuit direction averaged 39\% of those for the standard blocked experiment. There were no significant differences in the reduction of speed of pursuit after adaptation between the mixed and the standard blocked experiment, which averaged 90.8 and $87.8 \%$ of the control response, respectively. At least for adaptation of the direction of pursuit, our results parallel those for the perception of motion in which adaptation in one exposure has a strong influence on the adaptation caused during the next exposure (Wohlgemuth, 1911).

\section{Spatial specificity of adaptation}

As a first test of the spatial specificity of adaptation, we examined whether any effects of adaptation could be seen when the pursuit targets appeared in the opposite visual hemifield from where the adapting patch had been shown. In the experiments summarized above, both the control and adaptation blocks included pursuit targets that started either on the same (Fig. 3A) or opposite (Fig. $3 B$ ) side of the fixation position as the adapting stimulus. In contrast to the results when the pursuit stimulus moved through the same visual hemifield as the adapting stimulus (Fig. $3 C$ ), we did not observe adaptation of pursuit direction or speed for pursuit targets that moved through the same eccentricity in the opposite hemifield (Fig. 3D). The results of all 29 experiments are summarized in Figure 3, $E$ and F, where the mean and 95\% confidence intervals are shown as a function of the difference between the direction of motion of the pursuit target and the direction of the adapting motion. As before, these graphs plot the eye direction and speed in the adapting block relative to those in the control blocks. The data in Figure 2 have been replotted here (red symbols) for pursuit targets that moved through the same hemifield as the adapting stimulus. In contrast, the black symbols in Figure 3, $E$ and $F$, show that there was no evidence of adaptation when the pursuit target moved through the opposite visual hemifield.

As a more stringent test of the spatial specificity of the effect of motion adaptation on pursuit, we used adapting patches that
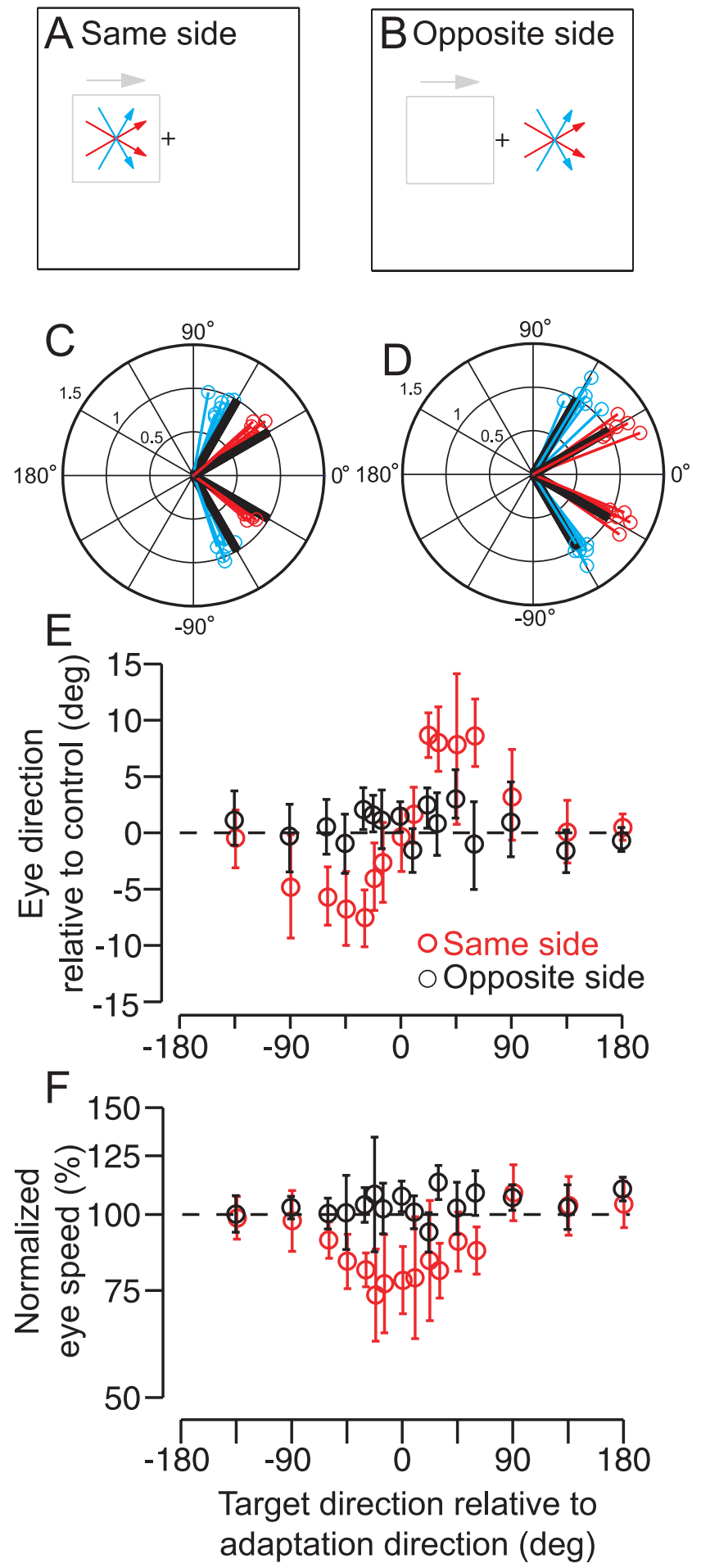

Figure 3. Failure of adaptation to generalize to the opposite hemifield from the adapting stimulus. $A, B$, Stimulus configuration. The light gray patch shows the location of the adapting stimulus, the gray arrow shows the direction of the adapting motion, the " + " indicates the fixation position, and the colored arrows show pursuit targets presented in the same $(A)$ or opposite $(B)$ hemifields from the adapting stimulus. $C, D$, Polar plots where each vector shows the normalized average eye velocity: black vectors show control responses, and colored vectors show responses from individual experiments in the adaptation block. The colors correspond to the arrows in $A$ and $B$, and the two plots show responses for pursuit targets that moved through the same $(C$ or opposite $(D)$ hemifield from the adapting stimulus. $E, F$, Summary of average effects of adaptation showing the normalized direction $(E)$ and speed $(F)$ in the adapting block as a function of the difference between the directions of motion of the pursuit target and the adapting stimulus. Red and black symbols show responses for pursuit targets in the same or opposite hemifield from the adapting stimulus. Error bars show $95 \%$ confidence intervals. 

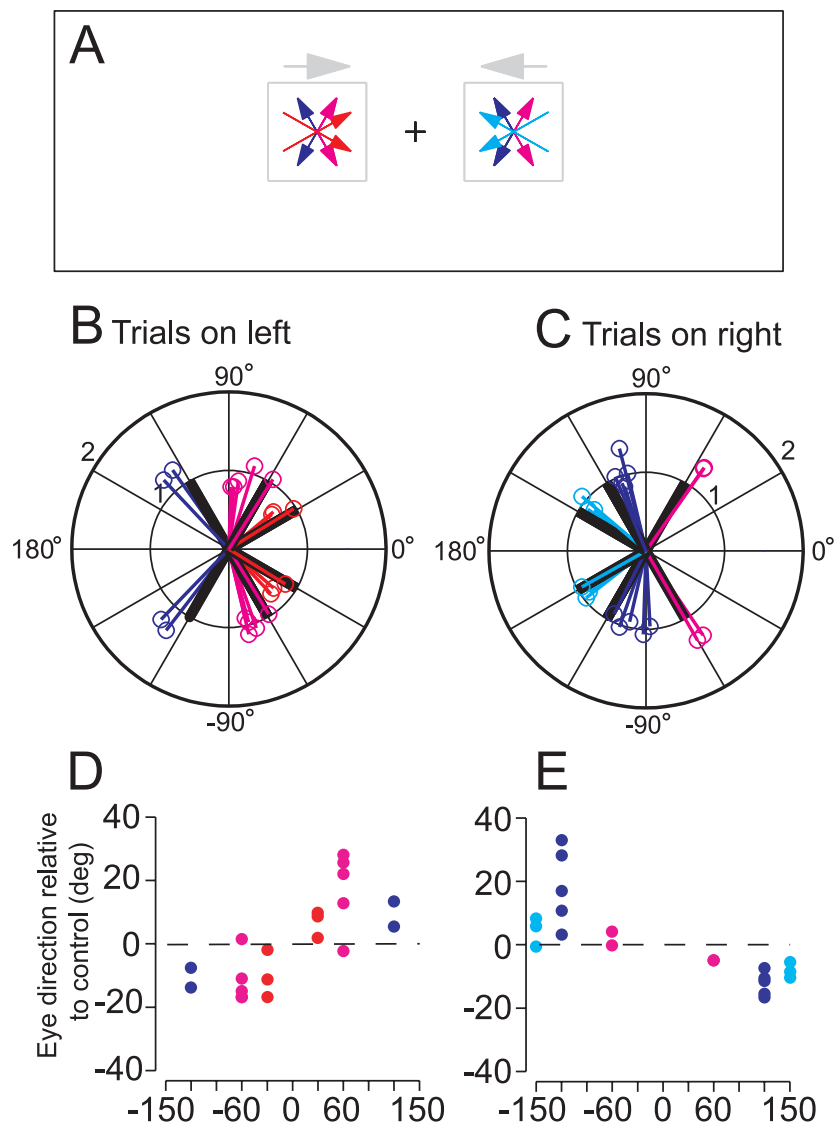

$\mathrm{F}$

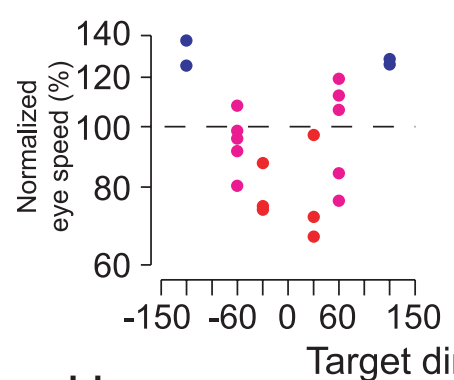

G
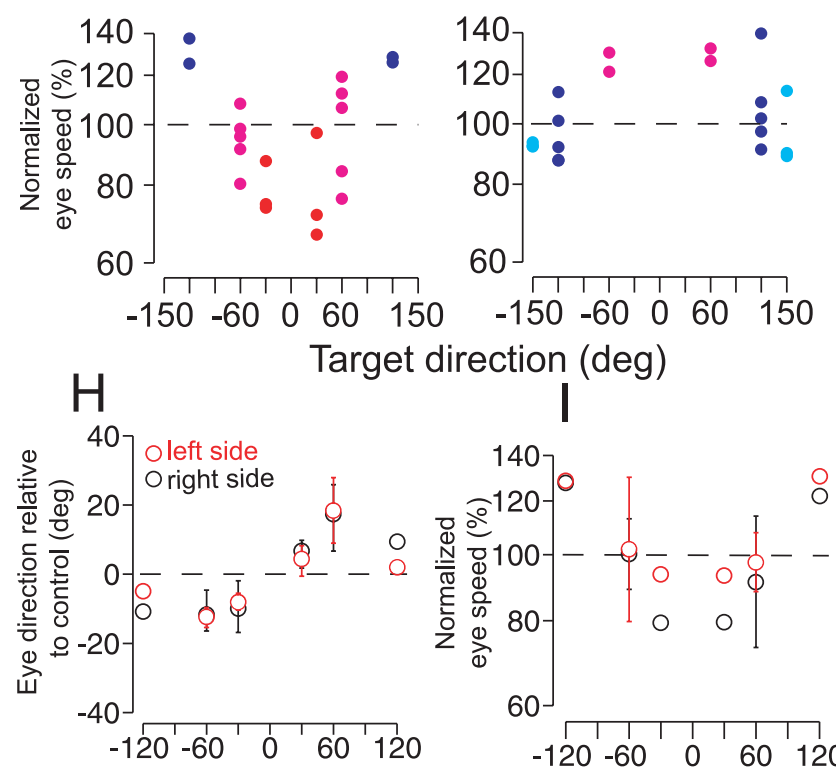

\section{Target direction relative to adaptation direction(deg)}

Figure 4. Effects of simultaneous adaptation with different directions of motion in the two visual hemifields. A, Stimulus configuration. The light gray patches show the locations of the two adapting stimuli, the gray arrows show the direction of the adapting motion in each patch, the " + " indicates the fixation position, and the colored arrows show pursuit targets presented in the two hemifields. $B, C$, Polar plots where each vector shows the normalized average eye velocity: black vectors show control responses, and colored vectors show responses from individual experiments in the adaptation block. The colors correspond to the arrows in $A$, and the two plots show responses for pursuit targets that moved through the left $(B)$ or right $(C)$ hemifield. $D, E$, Normalized eye direction is plotted as a function of the absolute target direction appeared simultaneously in the two visual hemifields and provided adapting motion in opposite directions. For the experiment in Figure 4, for example, we showed leftward adapting motion in the right visual hemifield and rightward adapting motion in the left hemifield (Fig. 4A). Subsequent pursuit targets moved in polar directions $-120,-60,-30,30,60$, and $120^{\circ}$ through location of the adapting patch in the left hemifield and in polar directions $-150,-120,-60,60,120$, and $150^{\circ}$ through the location of the adapting patch in the right hemifield (Fig. $4 B, C$; bold black vectors). The choice of directions represents a compromise between good coverage around the adapting direction in each hemifield and overlap in the directions of motion through the two hemifields.

The adaptation in pursuit for targets that moved through the visual field locations of the adapting patches in either hemifield was always appropriate to the motion of the relevant adapting patch. To document these effects, the two columns of Figure 4 summarize responses to target motion through the two hemifields. At each location on the abscissa in the graphs of Figure 4 $D-G$, each symbol shows results from an individual experiment. Here, the data are plotted as a function of the actual direction of target motion, so that the adapting direction corresponds to $0^{\circ}$ in Figure $4 D$ (and $F$ ) and $\pm 180^{\circ}$ in Figure $4 E$ (and $G$ ). Comparison of Figure $4, D$ and $E$, shows that eye direction was repelled from the direction of the adapting stimulus in each hemifield. It was rotated clockwise for pursuit targets with negative polar angles when the targets moved through the left hemifield and counterclockwise when the targets moved through the right hemifield (and in the opposite sense in each case for pursuit targets with positive polar angles). As a result, adaptation had different effects on the responses to pursuit target motion in a given absolute polar direction when the targets moved through different hemifields. For example, adaptation caused a negative shift for a direction of -120 on the left (Fig. $4 D$ ) and a positive shift for the same direction of target motion on the right (Fig. $4 E$ ). Adaptation of speed shows the same general effect. For targets in the left visual hemifield, which had rightward adapting motion, the eye speed was reduced for targets with a rightward component of motion and increased for targets with a leftward component of motion (Fig. $4 F$ ). The U-shaped curve suggested by Figure $4 F$ is contrasted by the upside-down U-shaped curve suggested by Figure $4 G$, which shows the eye speed of pursuit for targets that moved through the right hemifield after adaptation with leftward motion. Eye speeds were higher than control for targets with rightward components of motion and the same or slightly lower than control for targets with leftward components of motion. As with direction, there are examples of polar directions of motion in which the effect of adaptation on pursuit speed was opposite for the target motion through the two visual hemifields.

Figure 4, $H$ and $I$, confirm that the effects in the two hemifields are the same as those we documented in Figures 2 and 3 when the data are plotted as a function of the difference between the direction of motion of the pursuit target and the adapting direction.

$\leftarrow$

for pursuit targets that moved through the left ( $D$ ) or right $(E)$ hemifield. $F, G$, Normalized eye speed is plotted as a function of the absolute target direction for pursuit targets that moved through the left ( $F$ ) or right $(G)$ hemifield. In $D-G$, the colors of the symbols correspond to the arrows in $A$. $H, I$, Eye direction $(H)$ and speed $(I)$ as a function of the difference between the directions of motion or the pursuit target and the adapting stimulus. Red and black symbols show average responses across all experiments for targets that moved through the left and right visual hemifield, respectively. Error bars show $95 \%$ confidence intervals when there was enough data to compute them by bootstrapping. 
For pursuit targets in both the right (black symbols) and left (red symbols) hemifield, the adaptation causes eye direction to be repelled away from the adapting direction (Fig. $4 H$ ). The 95\% confidence intervals (error bars) indicate that the adaptation is statistically significant. The adaptation of eye speed is also similar for targets moving through the two visual hemifields, although there was very little reduction in eye speed for targets in the left hemifield and small numbers of trials precluded computation of $95 \%$ confidence intervals for some of the key points. We also note that simultaneous adaptation with opposite direction motion in the two hemifields caused eye speed to be considerably larger than control for targets that moved in the opposite direction from the adapting patch, in both hemifields. More data would be needed to determine the veracity of this apparent difference from the results with single adapting patches.

\section{Spatial generalization of the effects of adaptation}

We tested the spatial generalization of the adaptation effect more systematically in seven experimental sessions by presenting pursuit targets starting from multiple positions across the horizontal meridian (Fig. $5 A$ ) after adaptation with a $10 \times 10^{\circ}$ patch (Fig. $5 A$, white rectangle) located $10^{\circ}$ to the left (or right) of the fixation target. Pursuit targets moved in directions that were either +30 or $-30^{\circ}$ from horizontal, starting at positions chosen so that the targets would cross the horizontal meridian $250 \mathrm{msec}$ after motion onset at locations $-15,-10,-5,0,5$, and $10^{\circ}$ from the fixation point. The post-saccadic pursuit direction (Fig. $5 B$, open circles) and speed (Fig. 5C, open circles) underwent spatially specific adaptation. For pursuit targets that moved near the region of the adapting patch (target position $-15,-10$, and $-5^{\circ}$ on the abscissa), both the direction and speed of smooth eye velocity are below the horizontal dashed lines, indicating effects of adaptation. For pursuit targets that started central enough to cross the position of fixation or that moved through the opposite visual hemifield (target position 0,5 , and $10^{\circ}$ on the abscissa), there was little to no difference between the control and adaptation blocks in either the direction or speed of smooth eye movement.

To test the effects of adaptation of the central visual field, we conducted nine similar experiments with a $5 \times 5^{\circ}$ adapting patch that was centered at the position of fixation (Fig. $5 A$, gray rectangle). The effects of adaptation with the central patch were weaker than with the peripheral patch, but they show a complementary pattern of results (Fig. $5 B, C$; gray symbols). When the pursuit target moved through the central visual field, near the position where the adapting patch had been shown, both direction and speed were adapted relative to control. In contrast, when the pursuit target moved through peripheral positions, far from the location of the adapting patch, we did not see evidence of significant adaptation in either the direction or speed of smooth eye movement. We conclude that adaptation with a central a patch is specific to the retinal location of the adapting patch.

With a central patch, it was especially difficult for the monkeys to suppress smooth eye movements during adaptation. We therefore trained the monkeys over many experimental sessions to hold fixation with a central patch, decreasing the size of the fixation window over days until the monkeys were competent at performing the task. Even after training, the residual eye movements in the direction of the adapting patch were $1.01 \% \mathrm{sec}$, slightly larger than the $0.355^{\circ} / \mathrm{sec}$ found for trials with peripheral adapting patches. In neither case did average eye position over the course of the adaptation period differ by more than one-tenth of a degree from the center of the fixation position. We therefore conclude that any difference in the residual tracking movements
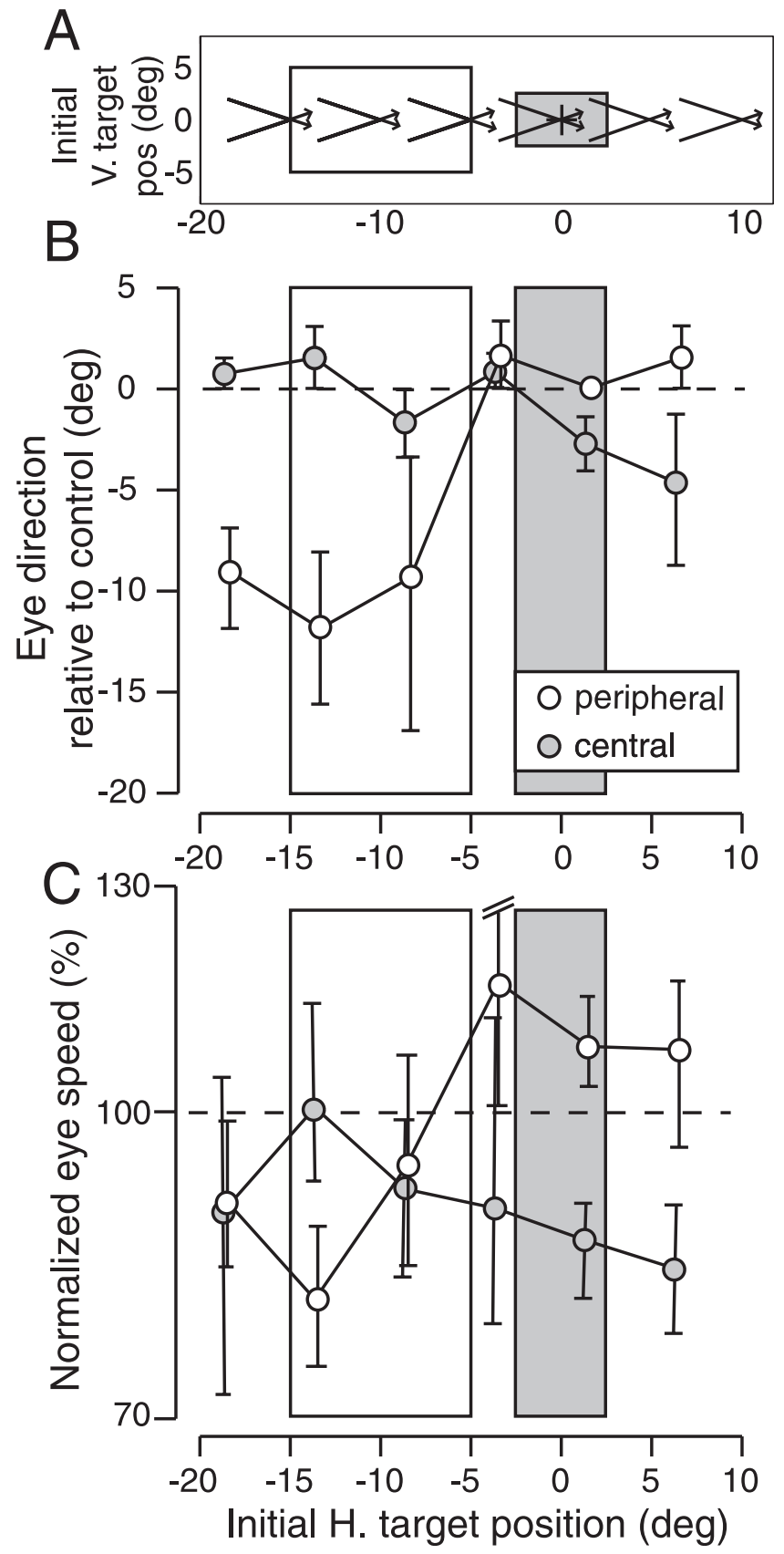

Figure 5. Spatial generalization of adaptation tested for peripheral and central adapting locations. A, Stimulus configuration. White and gray rectangles indicate the locations of the two adapting patches, used in different experiments, and arrows indicate the first $300 \mathrm{msec}$ of motion of the pursuit targets. $B, C$, Normalized eye direction $(B)$ and speed $(C)$ are plotted as a function of the starting location of the pursuit target along the horizontal axis. White and gray symbols show effects of peripheral and central adaptation, respectively. Error bars show $95 \%$ confidence intervals. White and gray rectangles indicate the region of the visual field covered by the peripheral and central adapting stimuli. Horizontal dashed lines show control values of eye direction and speed. For eye direction, negative values indicate that the response was repelled away from the direction of the adapting motion.

occurring during central patch presentation will have a minimal effect on the retinal velocity of the adapting patch $(<5 \%)$ and should not affect our results greatly.

\section{Time course of expression of adaptation}

For 1967 trials from 23 experiments in which the pursuit target direction was between 10 and $60^{\circ}$ from the adapting direction, we 


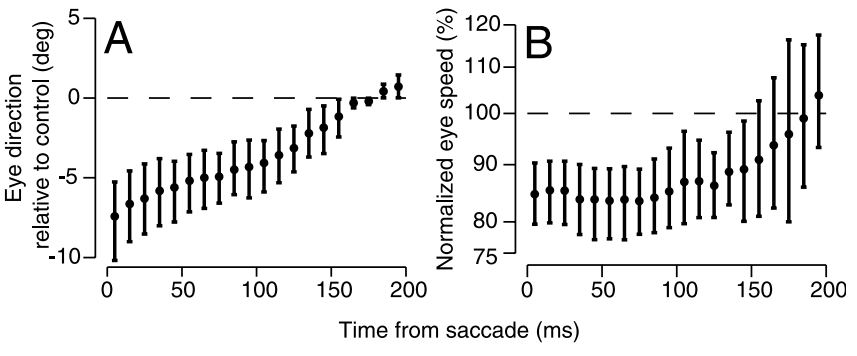

Figure 6. Time course of adaptation effects on post-saccadic pursuit. Normalized pursuit eye direction $(A)$ and speed $(B)$ are plotted as a function of time of the measurement relative to the saccade. Negative values of normalized eye direction indicate that the response was repelled away from the direction of the adapting motion. Error bars show $95 \%$ confidence intervals.

analyzed the effect of adaptation on the direction (Fig. 6A) and speed (Fig. $6 B$ ) of pursuit in $10 \mathrm{~ms}$ bins for $200 \mathrm{msec}$ after the end of the first saccade. The direction of pursuit (Fig. $6 \mathrm{~A}$ ) is plotted so that it has negative values if the response was repelled away from the adapting direction, showing that the expression of adaptation in pursuit recovers within 150-200 msec after the end of the first saccade. The speed of pursuit (Fig. $6 \mathrm{~B}$ ) returned to control over approximately the same time course. The short duration of the behavioral expression of adaptation does not imply that the underlying neural adaptation recovers as quickly. The initial position of the pursuit target was chosen so that the motion of the target would be presented at the same eccentric retinal location as the adapting patch, whereas after the first saccade, the target motion would be near the fovea, which had not been adapted. Therefore, our results agree well with the expectation that feedback about the errors in pursuit direction and speed would cause pursuit to recover within two latent periods after the first feedback indicated differences between target and eye velocity, despite persistent sensory adaptation. Because the configuration of target position and motion in our paradigm evoked early saccades and generally weak pre-saccadic pursuit, the pre-saccadic eye speed was low, and eye direction was poorly defined, thus precluding meaningful analysis of pre-saccadic adaptation effects in general. Furthermore, we found a small but significant difference in the average saccade latency in control and adaptation trials (202.9 vs $214.8 \mathrm{msec} ; p=0.013$; Student's $t$ test). To avoid confounds that might be caused by this difference, we have presented a conservative estimate of adaptation effects based on data aligned on the end of the saccades.

\section{Generalization of adaptation across target speeds}

In five experiments summarized in Figure 7 , we provided adapting motion at our standard speed of $16 \% \mathrm{sec}$ and tested pursuit with targets that moved in the adapting direction at speeds of 4,8 , $16,24,32$, and $48 \% \mathrm{sec}$. As reported previously, adaptation caused a reduction in post-saccadic eye speed for pursuit targets of the same speed as the adapting patch. The reduction was to a value that averaged $91.7 \%$ of control ( $p=0.07$; log-transformed Student's $t$ test). For other speeds of pursuit target motion, eye speed seemed to be repelled from target speed, much as it was in the direction domain. For target speeds of 4 and $8 \%$ sec, post-saccadic eye speed averaged 66.0 and $78.3 \%$ of control $(p<0.05$; logtransformed Student's $t$ test). For faster target speeds of 24 and $32 \%$ sec, post-saccadic eye speed averaged 104.2 and $107.2 \%$ of control ( $p=0.23$ and 0.05 ) For pursuit target motion at $48 \% \mathrm{sec}$, the adaptation was not present (although this speed was only tested for two experiments).

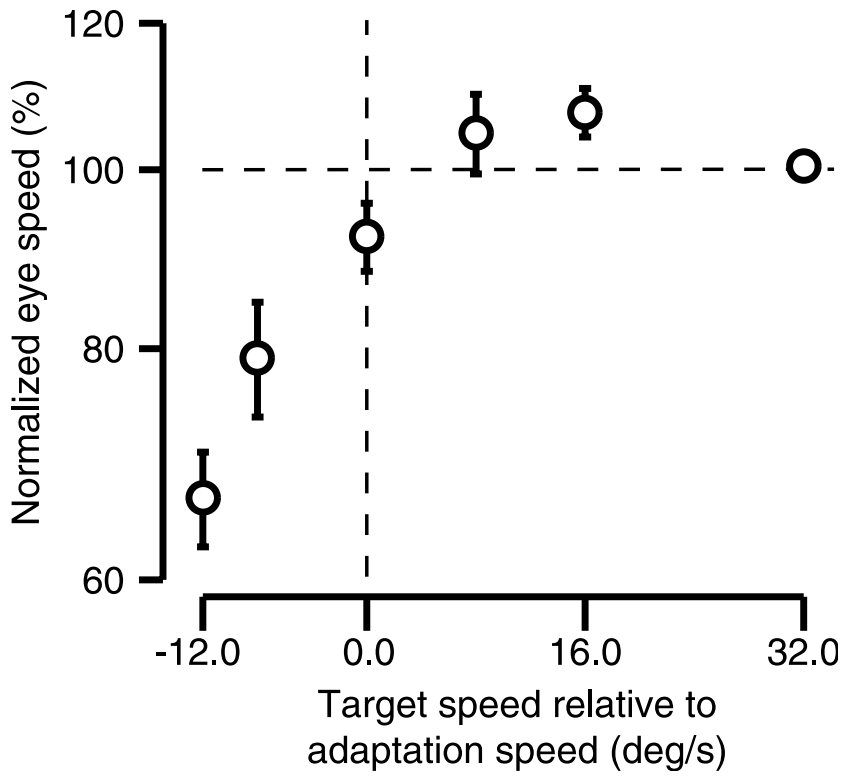

Figure 7. Effects of adaptation on the post-saccadic eye speed evoked by different speeds of target motion in the adapting direction. The graph plots normalized eye speed as a function of the difference between target speed and adapting speed. Targets moved at 4, 8, 16, 24, 32, and $48 \% \mathrm{sec}$, and the adapting stimulus always moved at $16 \%$ sec. Error bars show $95 \%$ confidence intervals. The target speed of $48 \% \mathrm{sec}$ (the point at $32 \%$ sec on the graph) was only tested in two experiments and therefore lacks error bars.
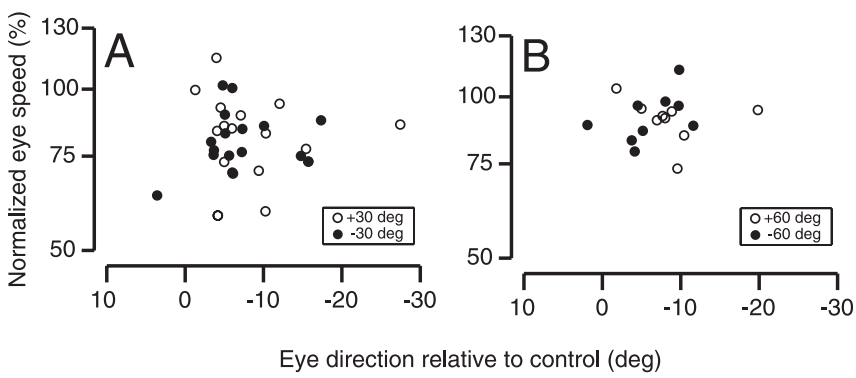

Figure 8. Absence of correlation in the magnitudes of the adaptations of eye speed versus eye direction. Data are plotted for each pursuit trial in which the direction of target motion was $30^{\circ}(A)$ or $60^{\circ}(B)$ from the adapting direction. Each point represents the average effect from $\sim 20$ trials in a single daily experiment. Negative values of normalized eye direction on the abscissa indicate that the response was repelled away from the direction of the adapting motion.

\section{Relationship of effects of adaptation on speed versus direction of pursuit}

To analyze whether the adaptation effects on the direction and speed of pursuit were coupled, Figure 8 plots the degree of adaptation of eye speed as a function of the adaptation of eye direction. Different points show averages of the effects in different experiments, and we made separate plots for pursuit targets that moved in directions that were $30^{\circ}(n=34)$ (Fig. $\left.8 A\right)$ or $60^{\circ}(n=20)$ (Fig. $8 \mathrm{~B}$ ) away from the adapting direction. There was no significant correlation between the magnitude of adaptation effects on speed and direction for either direction of pursuit target motion $\left(r^{2}<\right.$ $0.02 ; p>0.35)$. The same analysis of percentage of deviation in direction and speed in individual trials compared with the means for each experiment also did not reveal any correlation between the two effects of adaptation $\left(r^{2}<0.01 ; p>0.3 ; n=812\right)$.

\section{Computational analysis of adaptation of pursuit direction}

We have used a computer model to ask whether the effects of adaptation on the direction and speed of pursuit could be under- 
stood in terms of plausible estimates of the effect of adaptation on the population code for the direction of motion in area MT. Unfortunately, the available experimental data about the effect of adaptation on responses of MT neurons are disparate: previous recordings either provide data only about the effects of adaptation at the preferred and nonpreferred directions for each neuron (Petersen et al., 1985; Van Wezel and Britten, 2002; Kohn and Movshon, 2003) or have found qualitatively different results of adaptation on directiontuning curves depending on the adapting stimulus (Kohn and Movshon, 2004). In our analysis of direction adaptation, we therefore modeled four different types of effects of adaptation that have been found or suggested by recording studies on MT and V1 neurons.

In the first model, implemented as Equations 1 and 2, the response gain of MT neurons is decreased after adaptation (Petersen et al., 1985; Van Wezel and Britten, 2002; Kohn and Movshon, 2003). Adaptation extended to neurons with preferred directions near to the adapting direction, but the magnitude of adaptation decreased as a Gaussian function of the difference between the direction of the adapting stimulus and the preferred direction of the neuron. Adaptation was simulated as a multiplicative operation that scaled the Gaussian direction-tuning function by the same percentage for target motion in all directions. Neurons with preferred directions opposite or nearly opposite to the adapting direction were modeled as not being affected by adaptation (Van Wezel and Britten, 2002; Kohn and Movshon, 2003). Comparison of the red and black curves in the first column of Figure $9 A$ shows the effect of response gain adaptation on the direction-tuning curves for a family of model neurons. The amplitude of the adapted tuning curve (red) was smallest for the neuron with a preferred direction near the adapting direction and increased as the preferred direction of the neurons moved away from the adapting direction.

The effects of adaptation on a population of 10,000 model neurons with randomly chosen preferred speeds and directions can be appreciated qualitatively by examining the second and third columns of Figure $9 A$, each of which shows the population response to a given target motion. Each point plots the responses of one model neuron before (black) and after (red) adaptation, in which the position along the $x$-axis indicates the preferred direction of motion for the model neuron and the position along the $y$-axis indicates the response amplitude. When the direction of target motion was the same as the adapting direction (Fig. $9 \mathrm{~A}$, first column), adaptation reduces the amplitude of the population response, but the change in response is symmetrical around the direction of motion. As a result, the population vector is in the

D Attract
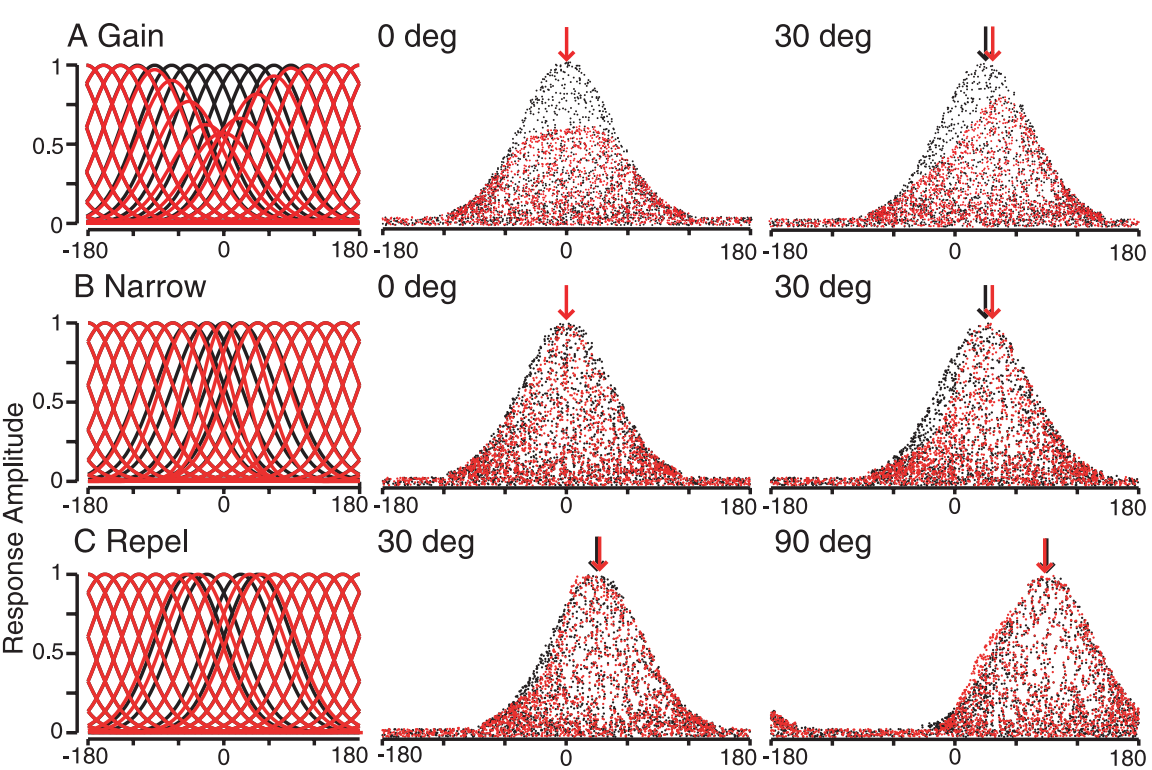

0 deg
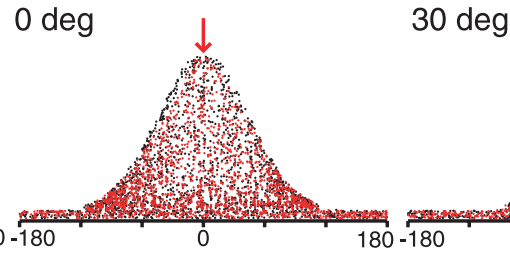

$30 \mathrm{deg}$

30 deg $\quad \quad \quad 90$ deg

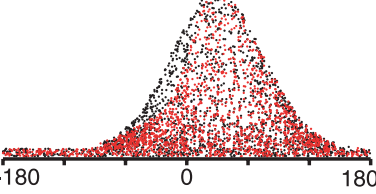
80 $15 \mathrm{deg}$
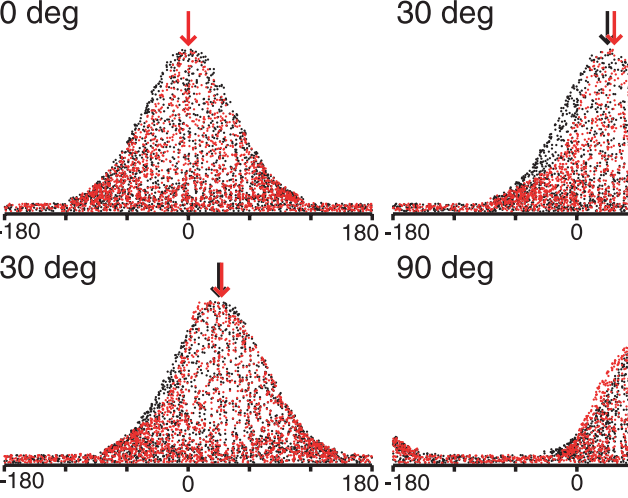

$\downarrow$

Figure 9. An explanation for the effects of motion adaptation in a population coding framework. $A-D$, Effects of different mechanisms of neural adaptation on the responses of model populations of MT neurons. Mechanisms of neural adaptation were: 政 , the leftmost columns of graphs show the direction-tuning curves of selected neurons before and after adaptation. The ins relative to the adapting direction. Each point shows the response of a different model neuron from a 10,000 neuron adaptation on the direction of pursuit responses to target motion in different directions. Black symbols show the mean and $95 \%$ nisms of neural adaptation represented by $A-D$.

same direction before and after adaptation (red and black arrows, respectively). In contrast, if the direction of motion of the pursuit target is in a polar direction that is $30^{\circ}$ from the adapting direction (Fig. $9 A$, third column), then adaptation depresses the response of model neurons asymmetrically on the side of the population response closest to the adapting direction. The direction of the population vector (red arrow) is repelled from the adapting direction, exactly as we observed in the initiation of pursuit. Similar logic has been used by others (Mather, 1980; Lee et al., 1988; Gilbert and Wiesel, 1990; Clifford et al., 2000; Clifford, 2002).

We fitted the 256 neuron model to the observed effect of adaptation on the direction of pursuit by allowing the amplitude, center, and SD of the adaptation function in Equation 2 to vary, as described in Materials and Methods. We obtained a best-fitting 
equation (Fig. 9E, black curve) that accounted for 95\% of the variance in the data using a maximum reduction of $33 \%$ of the response that was centered $3^{\circ}$ from the adapting direction, with the spread of adaptation characterized by a SD of $37^{\circ}$.

In the second model, implemented by Equations 1 and 3, the effect of adaptation was to narrow tuning widths (Kohn and Movshon, 2004). The best-fitting model (Fig. 9E, red curve) could account for $93 \%$ of the variance in the pursuit direction data by having a maximal effect for neurons with preferred directions $5^{\circ}$ from the direction of adaptation, where tuning widths were narrowed to $65 \%$ of their preadaptation values. The spread of adaptation was characterized by a SD of $28^{\circ}$. Inspection of the population responses from the 10,000 neuron model shows that narrowing the direction tuning has the same effect on the direction of the population vector as does adaptation by gain reduction, but without causing a large change in the amplitude of the population response. When the target direction is matched to the adapting direction (Fig. 9B, second column), the population response is symmetrically narrowed so that the direction of the population vector is unchanged. When the target direction is offset from the adapting direction (Fig. 9B, third column), the population response is altered asymmetrically, with a larger effect on the side closer to the adapting direction: the direction of the population vector is repelled from the adapting direction.

The third and fourth models were mirror images of each other, implementing adaptation by shifting the preferred direction of each neuron either toward (Kohn and Movshon, 2004) or away from the adapting direction, the latter similar to what has been found for orientation-tuning curves in V1 (Dragoi et al., 2000). Neither of these models of adaptation, implemented using Equations 1 and 4, did a good job of mimicking our data. For shifts in preferred direction away from the adapting direction, the best-fitting model accounted for only $18 \%$ of the variance in our pursuit direction data (Fig. 9E, cyan curve), using a maximum repulsion of $15^{\circ}$ that occurred for neurons with preferred directions $24^{\circ}$ from the adapting direction; the width of the adaptation effect was characterized by a SD of $16^{\circ}$ (Fig. 9 C, first column). For shifts in the preferred direction toward the adapting direction, the predictions of the model depended on how tightly the effects of adaptation were localized to the adapting direction. When adaptation effects were constrained to be localized near the adapting direction, the effect of attraction was essentially the opposite to that of repelling the tuning curves from the adapting direction. However, if we allowed adaptation to shift tuning by a maximum of $12^{\circ}$ over a larger range of angles (Fig. 9D), then the best-fitting model accounted for $44 \%$ of the variance.

The example population responses in Figure 9, $C$ and $D$, illustrate the reason for failure of the models in which adaptation caused attraction and repulsion of the preferred direction of MT neurons toward or away from the adapting direction. If changes in preferred direction are localized to neurons with direction tuning near the adapting direction (Dragoi et al., 2000; Kohn and Movshon, 2004), then the effects on the population response are biphasic, causing the shift in population response to be in one direction near the adapting direction but in the opposite direction far from the adapting direction. For the repulsion mechanism of adaptation (Fig. 9C), the population response was repelled from the adapting direction for target motion of a direction $30^{\circ}$ different from the adapting direction (middle column), in agreement with our data on adaptation of the direction of pursuit. However, the population response was attracted toward the adapting direction for target motion in a direction $90^{\circ}$ different from the adapting direction (right column), in contra- diction to our data. The model was never able to achieve a good fit to our data because of these two conflicting tendencies. The attraction mechanism of adaptation had the opposite problems, causing adaptation in the wrong direction for targets that moved in directions near the adapting direction and in the correct direction for targets that were further from the adapting direction.

If adaptation attracts or repels the preferred directions of MT neurons having a wider range of preferred directions, then the attraction model can predict the correct direction of adaptation in pursuit for targets that move in directions far from, but not near to, the adapting direction (Fig. 9D). When the direction of target motion was $60^{\circ}$ different from the adapting direction (right column), the population response was repelled from the adapting direction, in agreement with our data on the effect of adaptation on the direction of pursuit. However, when target motion was $15^{\circ}$ different from the adapting direction (middle column), the breadth of the adaptation function caused the same effects to occur on both sides of the population response. The population response broadened on both sides but did not change its vector average, predicting an absence of adaptation that we did not see in our data on pursuit. The repulsion model also failed to predict adaptation in pursuit for target motion in directions near the adapting direction and made the incorrect prediction that pursuit should be attracted toward the adapting direction for target motion in directions farther from the adapting direction.

\section{Computational analysis of adaptation of pursuit speed}

Two changes in the population response might contribute to the effects of adaptation of the speed of pursuit. First, there is abundant evidence that target speed is estimated by a neural implementation of Equation 6, where the presence of $\epsilon$ in the denominator means that adaptation will reduce eye speed if it also reduces the amplitude of the population response. Second, adaptation may have different effects on the speed tuning of MT neurons depending on the difference between the adapting speed and their preferred speeds. Because of the absence of neural data to predict the effect of adaptation on speed tuning, we have chosen not to extend our simulations along that axis. However, we can evaluate whether adaptation can be expected to cause changes in the speed of pursuit given the population responses for the different models in Figure 9 and reasonable values of $\epsilon$. In our data, the average effect of adaptation on the speed of pursuit when direction and speed are matched was $\sim 80 \%$. Previous models have predicted that the value of $\epsilon$ is $<25 \%$ of the normal population response. Equation 6 predicts that the population response must be reduced in amplitude to $<47 \%$ of control to match these constraints. For the four models we considered, the population responses to target motion in the adapting direction fell short of these predictions: the total population response was reduced to only $73 \%$ of the control amplitude for the gain model, to $90 \%$ of control for the narrowing model, and to $92 \%$ of control for the repelling model. Furthermore, the population response increased to $143 \%$ of control for the attraction model, in the wrong direction to account for the effect of adaptation on the speed of pursuit. We conclude that Equation 6 is adequate to account for the effects of adaptation on the speed of pursuit only if adaptation also affects the responses of MT neurons in a way that depends on the difference between the adapting speed and the preferred speed of the neuron.

\section{Discussion}

We have demonstrated appreciable and reproducible motion aftereffects on smooth pursuit eye movements. Exposure to small 
patches of coherently moving dots for several seconds results in marked changes in the direction and speed of pursuit. The changes depend on the relative locations, directions, and speeds of motion of the pursuit target and the adapting stimulus. For pursuit target directions near the adapting direction, the direction of pursuit is systematically repelled away from the adapting direction. When pursuit target directions are similar to the direction of the adapting patch, pursuit eye speed is depressed relative to control conditions in which the adapting stimulus is stationary. In what follows, we interpret our data in terms of brain mechanisms of population decoding, suggesting a common framework for understanding the processing of sensory signals for action and perception.

\section{Coordinate system for motion adaptation in pursuit}

The spatial specificity of motion adaptation in pursuit demonstrates the coordinate frame of adaptation is retinal and not motor. If adaptation were localized in motor circuits, it would have been expressed for pursuit of targets that moved across the parts of the visual field outside the site of the adapting motion. Thus, adaptation cannot be caused by a residual smooth eye movement left over from a larger response generated to suppress smooth tracking during presentation of the adapting stimulus. Furthermore, our results are distinct from those for pursuit motor learning, which shows partial generalization to targets presented even in the opposite hemifield (Chou and Lisberger, 2002) and is specific for the direction of target/eye motion rather than the direction of visual motion (Kahlon and Lisberger, 1996). Thus, motion adaptation in pursuit occurs in a sensory coordinate system, whereas pursuit learning occurs in an intermediate, sensory-motor coordinate system.

\section{Underlying effects of adaptation on neuronal populations}

Of the four possible effects of adaptation on the responses of MT neurons we tested in computer simulations, only changes in response gain or narrowing of tuning width could account for the effects of adaptation on the direction of pursuit. Neither attractive nor repellant changes in the preferred direction of tuning for MT neurons by themselves could account for the effects in our data. Kohn and Movshon (2004) found that a combination of response gain, narrowing, and attraction could account for the magnitude of perceptual effects of adaptation. Our study corroborates and extends their results by testing separately each of the mechanisms found in their study and comparing the predictions to quantitative data about the effect of adaptation on motor output. To account for both the magnitude and extent of adaptation effects on the direction of pursuit movements, only the neural adaptations comprising response gain and narrowing are critical. Although MT neurons do display attractive shifts of preferred direction after adaptation with sinusoidal gratings, there is preliminary evidence that this is not the case when the visual stimuli consist of the random dot patterns used in our study (Kohn and Movshon, 2004). We also note that a combination of changes in response gain and narrowing of tuning would work synergistically, allowing smaller neural adaptations of each type to account for the behavioral adaptation we have observed in the direction of pursuit.

Our models were based on the assumption that the response of each neuron contributes to the decoded estimate of target direction in the same way before and after adaptation. In principle, repellant shifts of preferred tuning away from the adapting direction still could account for our data if the value used to weigh each neurons response according to its preferred direction
( $\theta_{\text {pref }}$ in Eq. 5$)$ shifted with adaptation. As a consequence, tuning preferences would no longer be distributed uniformly across directions but would acquire a hole around the adapting direction. The effect on the vector-averaging decoding computation of Equation 5 would be to bias the estimate of target direction away from the adapting direction.

In all but the case just mentioned, the use of maximumlikelihood methods for estimating target direction from the population response would not have changed our conclusions. For populations of neurons that have direction tunings distributed equally across all directions, vector-averaging is mathematically equivalent to fitting a sinusoid to the population response, and maximum-likelihood is equivalent to fitting a tuning curve to the population response (Pouget et al., 2000). Each estimates the center of mass of the population response. If, in the repellant or attractive model of neural adaptation, we allow the "label" or weight in the population computation of each neuron to shift with preferred direction, then a maximum-likelihood computation would take account of the shift and would be resilient against the effects of neural adaptation, in disagreement with our pursuit data. These are important theoretical issues, but we do not want to give the impression that the exact decoding computation (or its neural implementation) is constrained by our report. We prefer to emphasize the value of the population coding and decoding framework for understanding the relationship between neural and behavioral expressions of sensory adaptation.

\section{Population decoding for speed versus direction}

In principle, the computation used to estimate target direction must be distinct from that used to estimate speed. The encoding of direction and speed by MT neurons is orthogonal in the sense that neurons have similar tuning curves for direction regardless of the speed of stimulus, and vice versa (Rodman and Albright, 1987). From the perspective of pursuit, estimates of target direction and speed have rather different consequences for motor programming. In the cerebellum and brainstem, the estimate of direction must specify the relative activity of different sets of neurons that drive the horizontal and vertical components of smooth eye velocity, whereas the estimate of speed will determine the overall level of activity in the different groups of neurons. At the very least, the computations used to decode speed and direction must assign different profiles of weights across the population of MT neurons. In our data, the absence of a correlation between the magnitudes of the effects of adaptation on pursuit speed and direction supports the idea that pursuit uses separate and mostly independent neural computations to estimate target speed and direction.

Our experiments and analysis leave open the question of how to account for the effect of adaptation on the speed of pursuit eye movements as well as the apparent repulsion of eye speed from the adapting speed. Because of the relative smallness of our data set on the effects of target speed along with the absence of knowledge of the effects of adaptation on the speed tuning of MT neurons, we have chosen to defer this issue for later, more extensive experiments and modeling.

\section{Comparison of adaptation effects for perception and action}

Our data show greater similarities to the perceptual motion aftereffects studied with dynamic stimuli than to those for static stimuli, such as the waterfall effect. The latter is thought to be an effect on low-level vision because it shows incomplete ocular transfer (Wohlgemuth, 1911) and is not produced by "secondorder motion” (Derrington and Badcock, 1985; Cropper and 
Hammett, 1997). Motion aftereffects tested on dynamic stimuli (Blake and Hiris, 1993) show almost complete ocular transfer (Raymond, 1993; Nishida et al., 1994) and can be induced by second-order motion (Ledgeway and Smith, 1994; Nishida and Sato, 1995). These and other results (for review, see Mather et al., 1998 ) indicate that tests with dynamic stimuli probe higher stages of visual processing. We think that the effects we see for pursuit are probing similar visual processing in the same brain areas that are responsible for motion aftereffects on dynamic stimuli.

The repellant adaptation effects on pursuit speed and direction mirror similar repellant effects in perceptual tests using tilt (Gibson, 1937), spatial frequency (Blakemore and Sutton, 1969), and image blur (Webster et al., 2002). Furthermore, perceived directions deviate away from the adapting direction in a way that appears quantitatively analogous to what we found for pursuit (Levinson and Sekuler, 1976). Although there is some heterogeneity of results concerning the perception of target speed after adaptation, the consensus of various tests show that adaptation affects perceived speed in a way similar to what we found (Carlson, 1962; Rapoport, 1964; Thompson, 1981; Smith, 1985). Thus, there is excellent agreement between the effects of motion adaptation on motor outputs and perceptual reports. We suggest that the locus of adaptation occurs early in the visual motion processing pathways, presumably in MT, so that the same adaptation affects both perception and action.

Adaptation can be a beneficial process for visual perception by keeping neurons sensitive to small changes in the baseline sensory stimulus across a wide range of baselines. Adaptation may serve a similar function for the perception of motion but would have potentially detrimental consequences for motion-based movements like pursuit, in which adaptation would degrade the ability to successfully match eye velocity to target velocity.

\section{A population decoding explanation for motion adaptation}

Our analysis has allowed us to take the next step beyond most previous physiology (Barlow and Hill, 1963; Vautin and Berkley, 1977; von der Heydt et al., 1978; Hammond et al., 1985, 1989; Petersen et al., 1985; Müller et al., 1999; Van Wezel and Britten, 2002; Kohn and Movshon, 2003) (but see Kohn and Movshon, 2004) and functional imaging experiments (Tootell et al., 1995; Huk et al., 2001) that understood adaptation based on responses of neurons to stimuli that moved in their preferred and nonpreferred directions (Sutherland, 1961). The strong causal linkage from visual responses in MT to pursuit eye movements has allowed us to create a broader framework for understanding adaptation based on population coding and decoding. The similarity of the motion aftereffects on pursuit and perception implies that our framework holds for both visual and movement systems, suggesting the existence of unifying principles of sensory coding and decoding for perception and action.

\section{References}

Addams R (1834) An account of a peculiar optical phaenomenon seen after having looked at a moving body. Lond Edin Philos Mag J Sci 5:373-374.

Albright TD (1984) Direction and orientation selectivity of neurons in visual area MT of the macaque. J Neurophysiol 52:1106-1130.

Bahill AT, Clark MR, Stark L (1975) The main sequence, a tool for studying human eye movements. Math Biosci 24:191-204.

Barlow HB, Hill RM (1963) Evidence for a physiological explanation of the waterfall phenomenon and figural after-effects. Nature 200:1345-1347.

Barnes GR, Asselman PT (1991) The mechanism of prediction in human smooth pursuit eye movements. J Physiol (Lond) 439:439-461.

Batschelet E (1981) Circular statistics in biology. London: Academic.

Blake R, Hiris E (1993) Another means for measuring the motion aftereffect. Vision Res 33:1589-1592.
Blakemore C, Sutton P (1969) Size adaptation: A new aftereffect. Science 10:245-247.

Carlson VR (1962) Adaptation in the preception of visual velocity. J Exp Psychol 64:192-197.

Chou IH, Lisberger SG (2002) Spatial generalization of learning in smooth pursuit eye movements: implications for the coordinate frame and sites of learning. J Neurosci 22:4728-4739.

Churchland MM, Lisberger SG (2001) Shifts in the population response in the middle temporal visual area parallel perceptual and motor illusions produced by apparent motion. J Neurosci 21:9387-9402.

Clifford CW (2002) Perceptual adaptation: motion parallels orientation. Trends Cogn Sci 6:136-143.

Clifford CW, Wenderoth P, Spehar B (2000) A functional angle on some after-effects in cortical vision. Proc R Soc Lond B Biol Sci 267:1705-1710.

Cropper SJ, Hammett ST (1997) Adaptation to motion of a second-order pattern: the motion aftereffect is not a general result. Vision Res 37:2247-2259.

Derrington AM, Badcock DR (1985) Separate detectors for simple and complex grating patterns? Vision Res 25:1869-1878.

Dodge R (1903) Five types of eye movement in the horizontal meridian plane of the field of regard. Am J Physiol 8:307-329.

Dragoi V, Sharma J, Sur M (2000) Adaptation-induced plasticity of orientation tuning in adult visual cortex. Neuron 28:287-298.

Dursteler MR, Wurtz RH (1988) Pursuit and optokinetic deficits following chemical lesions of cortical areas MT and MST. J Neurophysiol 60:940-965.

Georgopoulos AP, Schwartz AB, Kettner RE (1986) Neuronal population coding of movement direction. Science 233:1416-1419.

Gibson JJ (1937) Adaptation, after-effect and contrast in the perception of tilted lines. I. Quantitative studies. J Exp Psychol 20:453-467.

Gilbert CD, Wiesel TN (1990) The influence of contextual stimuli on the orientation selectivity of cells in primary visual crotex of the cat. Vision Res 30:1689-1701.

Goodale MA, Milner AD (1992) Separate visual pathways for perception and action. Trends Neurosci 15:20-25.

Hammond P, Mouat GS, Smith AT (1985) Motion after-effects in cat striate cortex elicited by moving gratings. Exp Brain Res 60:411-416.

Hammond P, Pomfrett CJ, Ahmed B (1989) Neural motion after-effects in the cat's striate cortex: orientation selectivity. Vision Res 29:1671-1683.

Huk AC, Ress D, Heeger DJ (2001) Neuronal basis of the motion aftereffect reconsidered. Neuron 32:161-172.

Judge SJ, Richmond BJ, Chu FC (1980) Implantation of magnetic search coils for measurement of eye position: an improved method. Vision Res 20:535-538.

Kahlon M, Lisberger SG (1996) Coordinate system for learning in the smooth pursuit eye movements of monkeys. J Neurosci 16:7270-7283.

Kohn A, Movshon JA (2003) Neuronal adaptation to visual motion in area MT of the macaque. Neuron 39:681-691.

Kohn A, Movshon JA (2004) Adaptation changes the direction tuning of macaque MT neurons. Nat Neurosci 7:764-772.

Komatsu H, Wurtz RH (1989) Modulation of pursuit eye movements by stimulation of cortical areas MT and MST. J Neurophysiol 62:31-47.

Kowler E, Steinman RM (1979) The effect of expectations on slow oculomotor control. II. Single target displacements. Vision Res 19:633-646.

Ledgeway T, Smith AT (1994) Evidence for separate motion-detecting mechanisms for first- and second-order motion in human vision. Vision Res 34:2727-2740.

Lee C, Rohrer WH, Sparks DL (1988) Population coding of saccadic eye movements by neurons in the superior colliculus. Nature 332:357-360.

Levinson E, Sekuler R (1976) Adaptation alters perceived direction of motion. Vision Res 16:779-781.

Lisberger SG (1998) Postsaccadic enhancement of initiation of smooth pursuit eye movements in monkeys. J Neurophysiol 79:1918-1930.

Lisberger SG, Westbrook LE (1985) Properties of visual inputs that initiate horizontal smooth pursuit eye movements in monkeys. J Neurosci 5:1662-1673.

Lisberger SG, Morris EJ, Tychsen L (1987) Visual motion processing and sensory-motor integration for smooth pursuit eye movements. Annu Rev Neurosci 10:97-129.

Mather G (1980) The movement aftereffect and a distribution-shift model for coding the direction of visual movement. Perception 9:379-392. 
Mather G, Verstraten F, Anstis SM (1998) The motion aftereffect: a modern perspective. Cambridge, MA: MIT.

Maunsell JH, Van Essen DC (1983) Functional properties of neurons in middle temporal visual area of the macaque monkey. I. Selectivity for stimulus direction, speed, and orientation. J Neurophysiol 49:1127-1147.

Mooney CZ, Duval RD (1993) Bootstrapping: a nonparametric approach to statistical inference. Newbury Park, CA: Sage Publications.

Müller JR, Metha AB, Krauskopf J, Lennie P (1999) Rapid adaptation in visual cortex to the structure of images. Science 285:1405-1408.

Newsome WT, Wurtz RH, Dursteler MR, Mikami A (1985) Deficits in visual motion processing following ibotenic acid lesions of the middle temporal visual area of the macaque monkey. J Neurosci 5:825-840.

Nishida S, Sato T (1995) Motion aftereffect with flickering test patterns reveals higher stages of motion processing. Vision Res 35:477-490.

Nishida S, Ashida H, Sato T (1994) Complete interocular transfer of motion aftereffect with flickering test. Vision Res 34:2707-2716.

Petersen SE, Baker JF, Allman JM (1985) Direction-specific adaptation in area MT of the owl monkey. Brain Res 346:146-150.

Pouget A, Dayan P, Zemel R (2000) Information processing with population codes. Nat Rev Neurosci 1:125-132.

Press WH, Flannery BP, Teukolsky SA, Vetterling WT (1988) Numerical recipes in C. Cambridge, UK: Cambridge UP.

Priebe NJ, Lisbeger SG (2004) Estimating target speed from the population response in visual area MT. J Neurosci 24:1907-1916.

Purkinje J (1820) Beiträge zur näheren Kenntniss des Schwindels aus heautognostischen Daten. Medicinische Jahrbücher des kaiserlichköniglichen öesterreichischen Staates 6:79-125.

Rapoport J (1964) Adaptation in the perception of rotary motion. J Exp Psychol 67:263-267.

Raymond JE (1993) Complete interocular transfer of motion adaptation effects on motion coherence thresholds. Vision Res 33:1865-1870.

Rodman HR, Albright TD (1987) Coding of visual stimulus velocity in area MT of the macaque. Vision Res 27:2035-2048.
Schrater PR, Simoncelli EP (1998) Local velocity representation: evidence from motion adaptation. Vision Res 38:3899-3912.

Smith AT (1985) Velocity coding: evidence from perceived velocity shifts. Vision Res 25:1969-1976.

Spigel IM (1960) The effect of differential post-exposure illumination on the decay of a movement after-effect. J Psychol 50:209-210.

Sutherland NS (1961) Figural after-effects and apparent size. Q J Exp Psychol 13:222-228.

Thompson P (1981) Velocity after-effects: the effects of adaptation to moving stimuli on the perception of subsequently seen moving stimuli. Vision Res 21:337-345.

Thompson P, Wright J (1994) The role of intervening patterns in the storage of the movement aftereffect. Perception 23:1233-1240.

Tootell RB, Reppas JB, Dale AM, Look RB, Sereno MI, Malach R, Brady TJ, Rosen BR (1995) Visual motion aftereffect in human cortical area MT revealed by functional magnetic resonance imaging. Nature 375:139-141.

Van Wezel RJ, Britten KH (2002) Motion adaptation in area MT. J Neurophysiol 88:3469-3476.

Vautin RG, Berkley MA (1977) Responses of single cells in cat visual cortex to prolonged stimulus movement: neural correlates of visual aftereffects J Neurophysiol 40:1051-1065.

Verstraten FA, Fredericksen RE, Grusser OJ, van de Grind WA (1994) Recovery from motion adaptation is delayed by successively presented orthogonal motion. Vision Res 34:1149-1155.

von der Heydt R, Hanny P, Adorjani C (1978) Movement aftereffects in the visual cortex. Arch Ital Biol 116:248-254.

Webster MA, Georgeson MA, Webster SM (2002) Neural adjustments to image blur. Nat Neurosci 5:839-840.

Wohlgemuth A (1911) On the after-effect of seen movement. Cambridge, UK: Cambridge UP.

Zeki SM (1974) Functional organization of a visual area in the posterior bank of the superior temporal sulcus of the rhesus monkey. J Physiol (Lond) 236:549-573. 